\title{
CAUSES AND SOLUTION OF APERIODICITY OF SUPERSONIC FLOW FIELD DOWNSTREAM OF A PROFILE CASCADE
}

The paper deals with problems of transonic flow field periodicity downstream of a profile cascade with finite number of blades. The origin of phenomena generating this aperiodicity in transonic wind tunnel is described. The solution of this problem by means of adjustable perforated tailboard is documented by results of optical measurements (interferometry, schlieren methods) on a turbine profile cascade consisting of six blades only. The conditions of formation of limit load regime are described.

Keywords: Turbine cascade, exit flow periodicity, transonic flow, porous tailboard, limit load

\section{Introduction}

One of the most important conditions for correctness and credibility of the results of blade cascade testing in wind tunnels is the inlet and the exit flow field periodicity. The periodicity of exit flow field is closely bound to number of profiles in the cascade. The optimal case is cascade with infinite number of blades (unfortunately impossible in aerodynamic experimental research). The lowest possible number of blades in a cascade ensuring the correct flow field parameters at least in the middle three channels of the finite profile cascade is recommended in ([1], [2]). However, these recommendations do not seem useful in the case of supersonic exit velocities because the exit flow field is affected by structures that come of free jet boundary behind the lateral profiles of the measured cascade. Aperiodic distributions of exit static and total pressure and exit flow angle along the cascade pitches as well as an increase of entropy are consequences of formation of the free jet boundary downstream the trailing edges at supersonic regimes of cascade operation. To prevent the origin of free jet boundary downstream of one of the lateral profiles, a perforated tailboard was used. A turbine profile cascade, which consists of six blades only, was used for checking of the effects of the perforated tailboard. The results of measurements with and without the perforated tailboard are compared.

\section{Free jet boundary and cascade exit supersonic flow field}

The exit part of flow field is affected by structures, which originate at free jet boundaries downstream of both outer blades of the measured cascade. It depends on the type of cascade, the shape of used profile, the stagger angle, the back pressure etc., whether the exit part of supersonic flow field in the cascade will be affected by flow structures that come either from free jet boundary behind the first or the last cascade profile, alternatively from both of them. The free jet boundaries arise on trailing edges of lateral profiles (see Fig. 1). The fan of expansion waves coming from trailing edge is followed by compression waves (due to the curvature of free jet boundary line), which are then again followed by a fan of expansion waves, etc. The process of alternating expansion and compression downstream the profile cascade is similar to the pseudo shock wave phenomenon [3]. Expansion and compression waves influence the exit flow field in different ways.

The expansion waves influence the flow field in the vicinity of the trailing edge. Flow on the surface exposed to the expansion waves is more accelerated and pressure conditions on the pressure and suction surface, which determine formation of near wake, are altered. Hence the base pressure behind each profile differs from blade to blade according to the angle of corresponding expansion wave. These pressure changes result in significant differences of flow structures in exit part of all affected interblade channels. At higher outlet velocities the aperiodic phenomena might promote earlier onset of the limit load regime. The limit load regime [4] is the fourth significant regime at operation of high - speed turbine blade cascades [5]. This regime determines conditions at which farther decrease of backpressure cannot affect flow development in the exit part of the profile cascade. The internal branch of exit shock waves either impinges on the trailing edge of the next profile or misses it completely. The supersonic exit flow deviation $\Delta a_{p s}$ increases. The condition of limit load can be determined according to the relation:

$$
\left|M_{2} \sin \beta_{2}\right| \geq 1
$$

\footnotetext{
* Martin Luxa ${ }^{1}$, Jaroslav Synac ${ }^{2}$, Pavel Safarik ${ }^{3}$, David Simurda ${ }^{1}$

${ }^{1}$ Institute of Thermomechanics ASCR, v.v.i., Prague, Czech Republic, E-mail: luxa@it.cas.cz

${ }^{2}$ R\&D dept., Skoda Power, Pilsen, Czech Republic

${ }^{3}$ Czech Technical University in Prague, Prague, Czech Republic
} 
where $M_{2}$ is the exit Mach number and $\beta_{2}$ is the exit flow angle (oriented to the rotor peripheral direction).

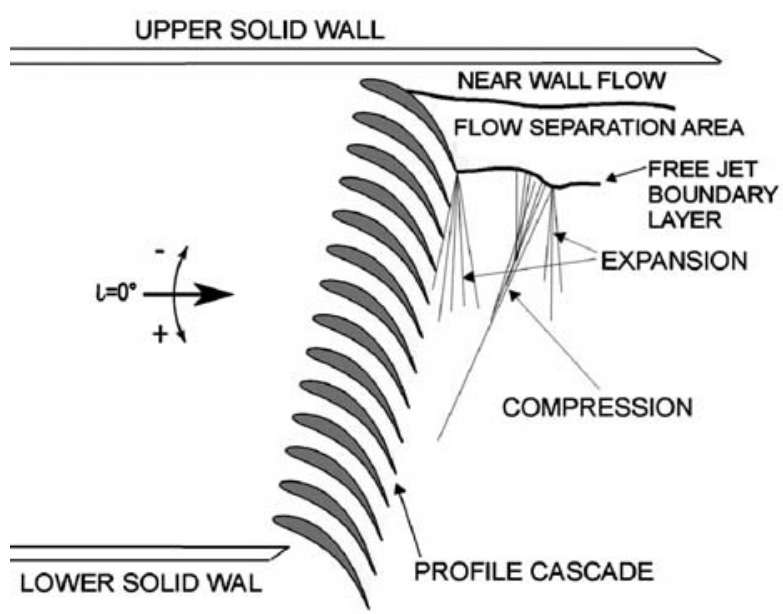

Fig. 1 Origin of aperiodic phenomena in the blade cascade exit flow field

The undesirable limit load regime, which is not caused by sufficiently small backpressure, can be reached in one or even in more interblade channels. The position of limit loaded channel (or channels) can be changed along the cascade according to the pressure condition downstream the cascade. The backpressure affects the formation of the free boundary layer and thus the mutual position of expansion and compression waves relative to the cascade. Figure 2 shows an example of exit flow field downstream a profile cascade affected by expansion waves in upper inter blade channels (here the limit load regime is seemingly reached); the flow field structures in exit parts of lower inter blade channels correspond to the real back pressure.

The compression waves combine together and form a shock wave. This undesirable shock wave can brake into the interblade channel and completely disturb the flow structures in the exit part of the channel. The interaction of these shock waves and the regular system of exit shock waves downstream the profile cascade is also inevitable. These phenomena, taking place due to formation and development of the free jet boundary behind a blade cascade with finite number of blades at supersonic regimes of cascade operation, result in aperiodic distributions of exit velocity and exit flow angle along the cascade pitches and increase of entropy.

Figures 3 and 4 show schlieren pictures taken at identical angle of incidence 1 but different back pressure. These figures document how the exit flow field may or may not be influenced by previously described phenomena. Due to lower back pressure and hence different exit flow deflection, the free jet boundary and the position of additional shock waves shifts and at lower backpressures may not influence the cascade exit flow field in the region of measurements at all (Fig. 4).

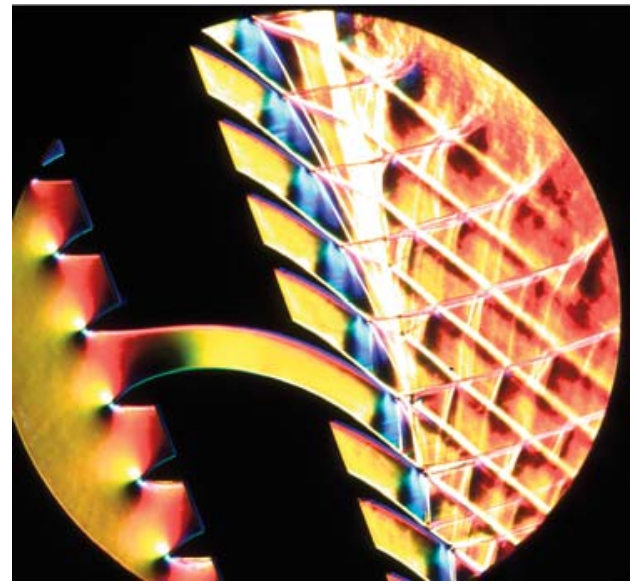

Fig. 2 The colour schlieren picture of the flow field in a profile cascade, $l=-30^{\circ}, M_{2 i s}=1.2$. The limit load condition is actually reached when $M_{2 i s} \sim 1.5$

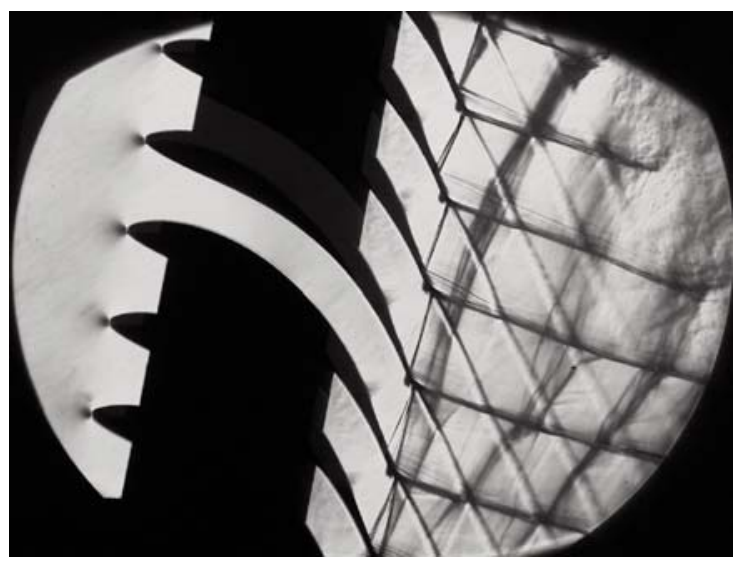

Fig. 3 The schlieren picture of the flow field in a profile cascade, $l=0^{\circ}$, $M_{2 i s}=1.402$. Additional shock waves appear in the exit flow field which is therefore disturbed and aperiodic

\section{Technique of ensuring periodicity of the supersonic flow field behind a blade cascade}

Various systems of perforated walls, solid, slotted or perforated tailboards, throttles etc. have been used in high speed experimental aerodynamic research to prevent the free jet boundary formation, e.g. [6]. In this study, configuration with only one perforated tailboard was used in the wind tunnel for $2 \mathrm{D}$ cascade testing. The perforated tailboard was mounted next to one of the lateral profiles (the suction side of this profile is outer suction side of the whole cascade), see Fig. 5. This way of tailboard design should prevent the origin of the free jet boundary and at the same time prevent the reflection of the system of exit shock waves back to the exit flow field. 


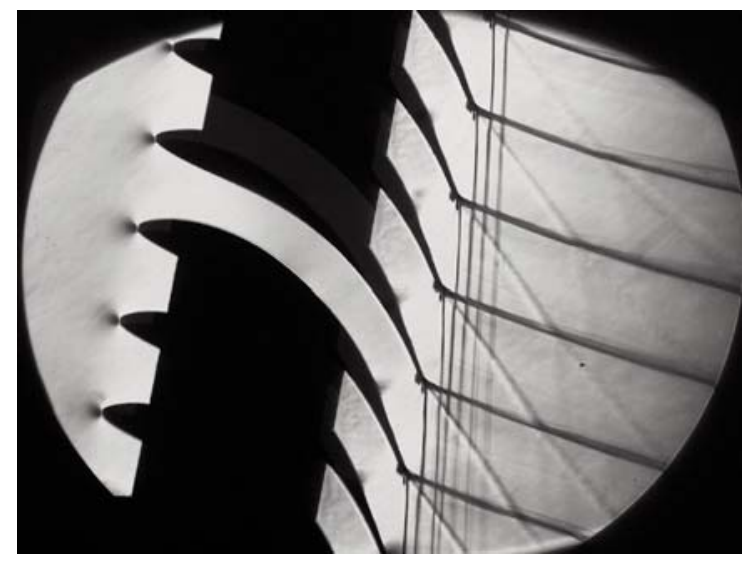

Fig. 4 The schlieren picture of the flow field in a profile cascade, $l=0^{\circ}$, $M_{2 i s}=1.828$. No additional shock waves visible, perfect flow field periodicity in exit part of interblade channels

The holes - solid wall ratio $R$ (or the open area ratio $R$ ) of the used perforated tailboard was chosen $R=0.5$. The value of this ratio was obtained from linearized theory of wave cancellation in perforated wind tunnels (the entropy changes are neglected and the compressibility equations are linearized) [7]. This ratio is independent of Mach number and shock intensity for the "non-reflection" case. If the area ratio $R$ of perforated walls is not selected correctly (i.e. $R=0.5$ ) a partial reflection of an impinging shockwave will occur. The pressure rise $\Delta p_{r}$ due to partial reflection of the primary shock wave can be determined from the relationship:

$$
\frac{\Delta p_{r}}{\Delta p_{s h}}=1-2 R
$$

where $\Delta p_{s h}$ is the pressure rise in the primary shock wave.

The value of angle between perforated tailboard and the plane of trailing edges $\alpha_{p s}^{\prime}$ was set according to the simple "empirical cosine rule", used for predicting of fluid outlet angle in design of turbo machines [8]. According to this rule, the value of exit flow angle $\beta_{2}$ is

$$
\beta_{2}=\arccos \frac{\Theta+2 r_{2}}{t},
$$

and thus

$$
\alpha_{p s}^{\prime}=\frac{\pi}{2}-\beta_{2}-\Delta \alpha_{p s}
$$

where $\Delta \alpha_{p s}$ is the tailboard angle correction for the exit velocities close to limit load regime and higher, $r_{2}$ is the trailing edge radius, $t$ is the pitch and $\Theta$ denotes the opening at the throat of the cascade.

Significantly complicated is the determination of proper value of tailboard angle $\alpha_{p s}^{\prime}$ in the case of exit flow conditions close to the limit load regime. It is necessary take into account the additional exit flow angle deviation or alternatively the supersonic flow deviation in the formula (4). The support of computational fluid dynamics methods (CFD) shows to be very effective and helpful in this situation.

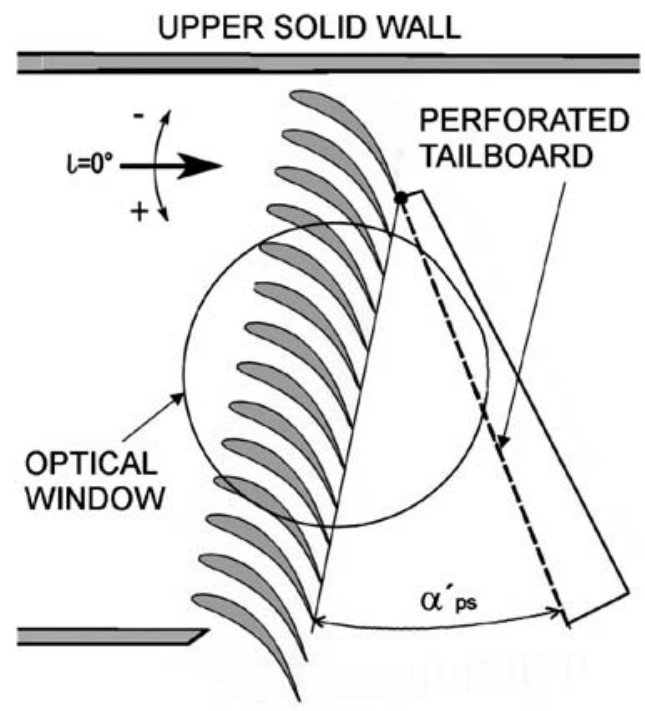

Fig. 5 An example of test section with installed tailboard

\section{Procedure and results}

The measured blade cascade represents a middle section of a long turbine rotor blade. The model blade cascade consists of six prismatic profiles only. The exit flow field and its periodicity were strongly affected in this case by structures which originate on the free jet boundary next to the suction side of the upper lateral profile [9].

The perforated tailboard was attached next to the trailing edge of the upper lateral profile (similarly to the situation in Fig. 5). The optimal value of angle between the tailboard and the plane of trailing edges $\left(\alpha_{p s}^{\prime}\right)$ was checked by colour schlieren technique in wide range of incidence angles, exit Mach numbers, angles $\alpha_{p s}^{\prime}$, etc. No tailboard angle correction $\Delta a_{p s}$ was necessary for exit velocities considerably smaller than those corresponding to the limit load regime. It was found out that the optimal value of the angle $\alpha_{p s}^{\prime}$ corresponds to the rule (3). An example of flow field with $\Delta \alpha_{p s}=0^{\circ}$ and $\Delta a_{p s}=-3^{\circ}$, respectively, is shown in Fig. 6 .

The optical methods were applied (interferometry, colour schlieren method and schlieren method in Toepler configuration) and results were compared with results of measurements without the tailboard. The experiments were carried out for isoentropic exit Mach numbers and incidence angles in the range.

The interferograms in Fig. 7 show different structures in the exit part of flow field in the case without tailboard (left) and with it (right). 


\section{COMMNICOIIONS}
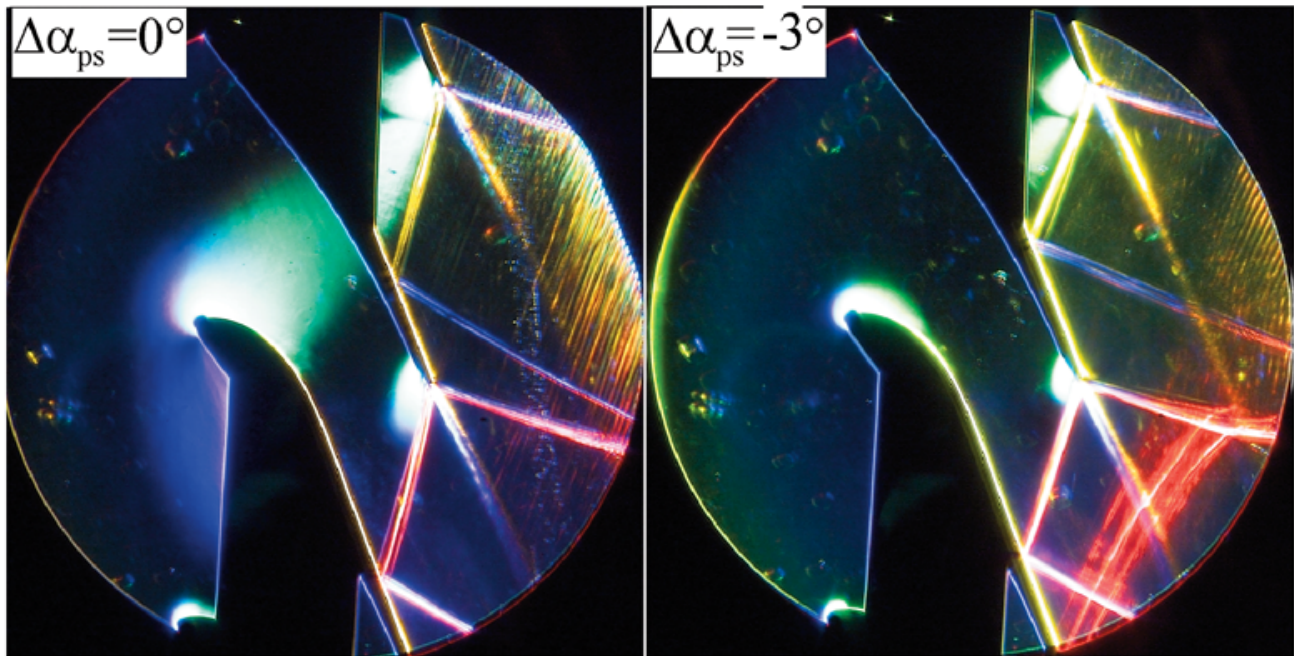

Fig. 6 The colour schlieren pictures taken for two different angles of perforated tailboard $\alpha_{p s}{ }_{p s}$, isoentropic exit Mach number $M_{2 i s}=1.5$, incidence angle $\iota=+30^{\circ}$
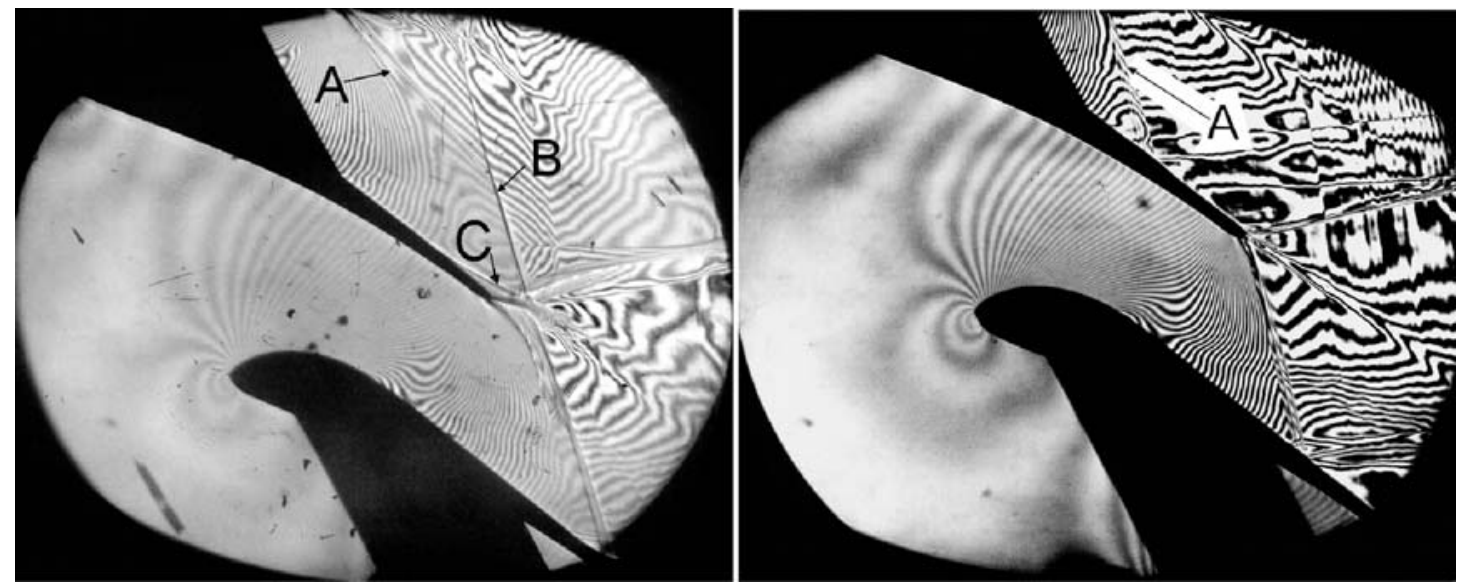

Fig. 7 Interferograms; isoentropic exit Mach number $M_{2 i s}=1.5$, incidence angle $l=0^{\circ}$; flow field affected by structures formed on the free jet boundary (left); different, periodic exit flow field in the arrangement with perforated tailboard (right)

The left interferogram shows that the regular periodic flow structures are completely disturbed as a consequence of expansion waves and shock waves originating on the free jet boundary. Due to expansion waves influencing flow on the suction side before the leading edge, the exit flow is significantly deflected. This can be seen on the interferograms as an increased obliqueness of exit shock waves internal branch of which (A) completely misses suction side of the neighbouring blade. This deflection is so intense that the near wake is formed before trailing edge on the suction side (B). The additional shock wave (C) goes through the wake downstream the upper profile and have also influence on the flow structures in the middle inter blade channel. The situation is completely different in the right part of Fig. 7. The exit part of the flow field is substantially more periodic, both visible internal branches of exit shock waves are parallel. Only small disturbances, caused by the perforated tailboard, appear on the right side of the interferogram near the tailboard.

The isoentropic Mach number $M_{i s}$ distributions on the profile suction side at incidence angle $l=+30^{\circ}$ and different exit isoentropic Mach numbers $M_{2 i s}$ are shown in Fig. 8. The effect of the structures coming from the free jet boundary is evident in the upper diagram.

\section{Conclusions}

Phenomena originating on the free jet boundaries behind the lateral profiles of a blade cascade operating at transonic regimes pose a problem since these phenomena significantly influence flow 
field at the exit of the blade cascade and farther downstream. Thus the qualitatively and quantitatively altered flow field largely differs from the case of periodic flow field and exhibits increased level of produced entropy. Therefore, periodicity of the flow field at the exit of blade cascades must be ensured. The solution of problem with periodicity of the supersonic flow field downstream of a profile cascade lies namely in the necessity to prevent the origin and development of the free jet boundary downstream the cascade. To achieve this, adjustable perforated tailboard was used in this research. The analysis of optical measurements proves noticeable differences in the flow field structures in exit parts of inter blade channels and downstream. The results of optical measurements with the tailboard confirm substantial exit flow field periodicity improvement.
Nevertheless, successful application of the tailboard depends on correct choice of the perforation ratio and proper adjustment of the tailboard angle corresponding to the exit flow angle behind an infinite blade cascade. The shortcoming of using the perforated tailboard is origin of small disturbances caused by the tailboard perforation. These disturbances probably cause an increase in level of turbulence in the vicinity of the tailboard. However, intensity of these disturbances and its influence on the flow field in the described experiments are yet to be investigated.

\section{Acknowledgement}

The research was supported by the Czech Science Foundation under the grant No.GAP101/10/1329.
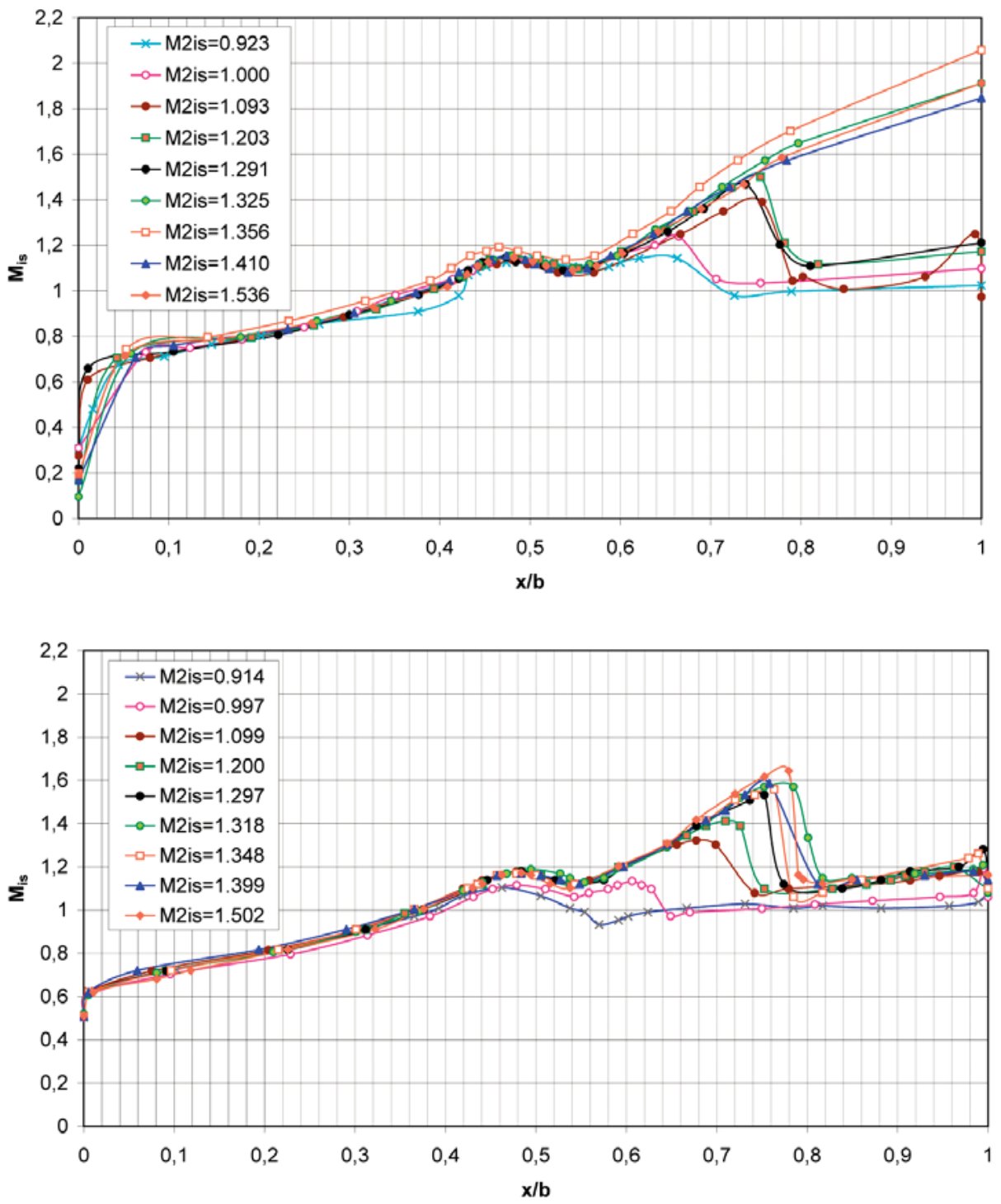

Fig. 8 Isoentropic Mach number distribution on the suction side on the profile. Isoentropic exit Mach number $0.914 \leq M_{2 i s} \leq 1.536$, incidence angle $\imath=+30^{\circ}$; upper diagram: Mach number distribution affected by structures formed on the free jet boundary; lower diagram: Mach number distribution in the arrangement with perforated tailboard 


\section{COMMNICOIIIONS}

\section{References}

[1] GUKASOVA, E. A.: Aerodynamiceskoje soversenstvovanije lopatocnych apparatov i gazovych turbin [Aerodynamic Design of Turbomachines]. Moskva, 1960

[2] STRAKA, P.: Numericka simulace pri podpore experimentalniho vysokorychlostniho aerodynamickeho vyzkumu proudeni v primych lopatkovych mrizich [Numerical Simulation as a Support of Experimental High-speed Aerodynamic Research in Straight Blade Cascades]. Ph.D. Thesis, Prague, 2010

[3] CROCCO, L.: One Dimensional Treatment of Steady Gas Dynamics. In: Fundamental of Gas Dynamics, ed. H.W. Emmons, High Speed Aerodymanics and Jet Propulsion, vol. III., Princeton U.P., 1958

[4] LUXA, M., SIMURDA, D.: Opticka mereni na mrizi TR-P-2 [Optical Measurements on Cascade TR-P-2]. Research report IT CAS v.v.i., No. 1472/11. Prague, 2011

[5] SAFARIK, P., LUXA, M., PICMAUSOVA, H., SIMURDA, D.: On Measurement and Evaluation of Flow Conditions at Limit Load of Turbine Blade Cascade. XXI Biannual Symposium on Measuring Techniques in Turbomachinery, Valencia, 2012.

[6] STARKEN, H.: A New Technique for Controlling the Exit Flow Periodicity of the Supersonic Cascades. Measuring Techniques in Transonic and Supersonic Cascades and Turbomachines, ed. A. Bolcs and T. Fransson, Zurich, 1977

[7] GOETHERT, B. H.: Transonic Wind Tunnel Testing. Oxford, 1961

[8] DIXON, S. L.: Fluid Mechanics and Thermodynamics of Turbomachinery, Amsterdam, 1998

[9] LUXA, M., SIMURDA, D.: Opticka mereni na transsonicke mrizi [Optical Measurements on Transonic Cascade]. Research report IT CAS v.v.i., No. 1476/11. Prague, 2011 